\title{
Digital Coaching - An Exploratory Study on Potential Motivators
}

\author{
József Mezei \\ Åbo Akademi University \\ jozsef.mezei@abo.fi
}

\author{
Anna Sell \\ Åbo Akademi University \\ anna.sell@abo.fi
}

\author{
Pirkko Walden \\ Åbo Akademi University and \\ Institute for Advanced \\ Management Systems Research \\ pirkko.walden@abo.fi
}

\begin{abstract}
The objective of our study is to explore the importance of different sets of functionalities in a digital coaching system. Digital coaching is defined as systems providing the user with actionable advice and feedback to reach fitness goals. From previous research we identify five sets of functionalities likely to be important in the digital coaching context: mental support, exercise programs, goal setting, feedback and social functionality. We employ Fuzzy-set Qualitative Comparative Analysis to understand users' opinions of digital coaching. Our results highlight the importance of exercise programs and goal setting functionality, whereas feedback and social functionality are surprisingly not so important. Some gender-related differences emerge.
\end{abstract}

\section{Introduction}

We define digital coaching as software solutions providing the user with actionable advice and feedback to reach fitness goals [13]. Digital coaching differs from other sports technology which provide data and visualizations of the user's activities, but do not suggest actions or give personalized plans. Central to digital coaching is that it is adaptive to the individual's needs, context-aware and timely [14]. Digital coaching has been investigated also in other contexts, e.g. to reduce procrastination in an educational setting [29] and as a self-help intervention to recover from binge eating [3].

In this study we investigate which potential functionalities of digital coaching are predictors of a positive attitude towards a digital coaching system. We utilize five sets of functionalities based on previous literature on traditional coaching, digital coaching as well as motivation and adherence to physical activity: mental support, exercise program, goal setting, feedback and social functionality. Our focus is on coaching for wellness and everyday physical activity, rather than for competition.
A general assumption present in many statistical methodology-based analyses is symmetry. A symmetric relationship implies that it is both necessary and sufficient. While this assumption can be valid in many situations, different complex phenomena require a different type of analysis. For example, one cannot assume in any situation that while a characteristic A of a service implies positive perception of the service, the lack of characteristic A would result in negative perception. Another important property, not always addressed by traditional methods, is equifinality: different distinct co-existing explanations exist to understanding a complex phenomenon. In order to allow for modelling these properties in our analysis to understand users' opinion on digital coaching, we will utilize a configurational methodology, Fuzzy-set Qualitative Comparative Analysis (FsQCA) [20]. FsQCA is a novel method which offers certain benefits in comparison with e.g. regression-based methods, making it suitable for studying user attitudes in technology studies.

The rest of the paper is structured as follows. In Section 2, a brief literature review is presented on different important functionalities of digital coaching with additional motivation on utilizing Fuzzy-set Qualitative Comparative Analysis for the research problem. The methodology, data preprocessing and descriptive analysis are presented in Section 3 with the main results included in Section 4. The results are discussed in Section 5, and finally some conclusions are presented in Section 6.

\section{Background}

In the following we provide the motivation for the five sets of functionalities we focus on in this study. A sixth set of functionalities, data privacy, was considered to be included in the study, but was omitted from further analyses as being ranked as important by virtually all respondents.

Mental support refers to functionalities aimed at keeping the user confident and focused. It is adapted from the Coaching Behavior Scale for Sport (CBS-S), a 
scale derived from qualitative research with coaches and athletes, used to assess the behavior of traditional sports coaches $[2,33]$. To fit the non-competitive context, we omitted two variables related to mental preparation for competition. Emphasizing positive reinforcement and encouragement have been found to be related to better psychosocial outcomes in coaching [27].

In addition, Exercise program has its origins in the CBS-S, there called Physical Training Planning; again we omitted a variable meant for a competitive context. Providing a tailored exercise program is, in a wider perspective, one of the key benefits of digital coaching; it becomes possible and cost-efficient to cater individualized exercise program also to persons who do not have the possibility to employ a personal trainer. Adherence rates to exercise programs are increased in supervised programs [18]. Further, there is evidence that adherence to a physical training program is greater when the individual believes that the program will be effective (the outcome expectancy) [18], that she is able to follow the requirements of the program (the efficacy expectancy) and also when she has a higher level of selfefficacy regarding the program [18]; individualized programs are more likely to support both feelings of self-efficacy and efficacy.

Goal setting is linked to exercise adherence, especially process goal setting as opposed to outcome goal setting [7, 32]. We measure goal setting in accordance to the CBS-S. Goal setting can aid in exercise adherence e.g. through breaking goals down to manageable parts and through adapting goals to the individual's situation; for example, goals are likely to change with age [4]. Both of these are suitable functionalities to a digital coach, where the individual's data is refined to personalized goals. Kulyk et al. [15] describe a suitable personal goal to be "challenging and reachable, step-by-step, within a set period of time" in their study on digital coaching systems.

Feedback is an important part of a coaching process. Feedback during exercise has been found to increase adherence to an exercise program [7]. Feedback should ideally be interactive, including both self-assessment by the individual and an action plan [12]. Kulyk et al. [15] evaluate feedback functions in a digital coaching system and confirm the importance of timeliness and tailoring. In our study, the feedback dimension is roughly equivalent to the Technical skills dimension in the CBSS.

Finally, the social functionality dimension represents functions connecting the user in different ways to other users, for example to encourage each other, to compare with each other and to gain inspiration from other users' habits and results. Perceived social support has been found to be an important factor associated with exercise participation both among older adults [21] and youth [8]. Moreover, previous research suggests that social functionality in software offers users gratification through being connected with other users [6]. We found further support for this in our own research; in a focus group study, social functionality emerged as highly important for the participants, heightening their motivation to adhere to exercise [25].

These five dimensions are relevant to understanding digital coaching. Traditional statistical analyses focus on independent variables and crisp cut-off values, whereas we believe that we can understand the users better by analyzing combinations of the dimensions. Therefore, we employ Fuzzy-set Qualitative Comparative Analysis, which enables a more nuanced representation of user attitudes. In the following we work through FsQCA in more detail.

\subsection{Fuzzy-set Qualitative Comparative Analysis}

In this section we briefly summarize the main advantages of Fuzzy-set Qualitative Comparative Analysis (FsQCA) and highlight the main differences compared to traditional statistical techniques. The main methodological basis of FsQCA originates from the theory of fuzzy sets introduced by Zadeh [35]. Fuzzy set theory recognizes that the restrictions of utilizing crisp sets (i.e. everything either fully belongs to a set or does not belong at all) does not offer a sufficiently refined representation in many (decision) problems. By utilizing a degree of membership belonging to a set, fuzzy set theory-based models allow for considering objects as belonging to different classes at the same time to varying degree. While originally fuzzy set theory has been applied originally in engineering problems by creating rule-based systems, in recent decades it has found many applications in different domains of the social sciences and business [28]. One of the widely used approaches, originally developed to address problems in the political sciences, is Fuzzy-set Qualitative Comparative Analysis (FsQCA) introduced by Ragin [20]. This approach uses Boolean algebra and various algorithms based on logical operations in combination with fuzzy sets to reduce a large number of complex causal conditions characterizing a phenomenon into a set of configurations that lead to a certain outcome [9].

\subsubsection{Comparing FsQCA and regression based models}


There are several important differences between FsQCA and traditional statistical techniques used for assessing the strength, direction and significance of relationship among variables of interest [16]. While traditional methods, such as linear regression, aim to measure the effect of (several) independent variables on the outcome, FsQCA attempts to identify different combinations of the independent variables (termed as conditions in this context) that, when simultaneously having high or low value, result in the given outcome [23]. The two main assumptions that are central in most of the traditional methods that are not assumed when utilizing FsQCA are casual symmetry and linearity [5]. While the assumption of casual symmetry is reasonable in many information systems phenomena, there are cases when we can encounter asymmetric relationships. For example, the result of traditional correlation analysis could suggest that high level of need for mental support in digital coaching is associated with willingness to adopt digital coaching. However, in reality we can encounter also a large number of users belonging to the following groups: (i) users who do not perceive a need of mental support but are intent to adopt digital coaching; (ii) users who perceive a need of mental support but are not willing to adopt digital coaching. Understanding the behavior of users with traditional models can be very challenging. Linearity refers to the traditional assumption of various statistical techniques modelling the hypothesized relationships using linear functions and extracting contributions of individual predictors. In contrast, FsQCA focuses on configurations of individual conditions and attempts to capture the effect of these complex combinations on a dependent variable. Additionally to these considerations, FsQCA strongly supports the equifinality principle: the typical outcome of the analysis results in several possible distinct configurations that provide different explanations on why an individual case results in the scrutinized outcome [31].

\subsubsection{FsQCA in technology adoption studies}

In recent years, FsQCA started to be utilized in studies investigating users' adoption behavior [34], in particular in the context of mobile services [30]. In this context, the complex patterns of causal interrelationships between user and service characteristics and the user's attitude towards the services is found to be appropriately modelled by FsQCA. Additionally to the benefits mentioned above, a frequently utilized extension/interpretation of FsQCA by Fiss [10] allows for differentiating between necessary and sufficient conditions. A prime example of a relevant contribution is presented by Yueh et al. [34], where they attempt to identify the relevant causal antecedents of a service's perceived work performance improvement. The study extends the understanding gained from performing traditional structural equation modeling and identifies the different configurations that explain the differences on how performance expectancy, facilitating conditions, social influence and usage behavior contribute to improved work performance perception.

\section{The study}

According to the discussion in the previous section, the starting point of our empirical study is the assumption that perceived importance of digital coaching services should be approached by utilizing a method allowing for the representation of non-linear and asymmetric relationships. In order to achieve this goal, as the first step, based on the identified set of potential antecedents of digital coaching usefulness, a questionnaire was designed. The empirical data was collected through a self-administered questionnaire in 2018. The questionnaire was available online for students participating in information systems courses at Åbo Akademi University ( $\AA A U)$ in Turku, Finland and at Linnaeus University (LU) in Kalmar, Sweden. The sample was a convenience sample, based on our access to students' email addresses. The number of completed, valid responses was 138 , with $30 \%$ of them studying at the Finnish University. The average age of the sample is 28.01 years (27.93 at $\AA \mathrm{AU}$ and 28.03 at LU) with median value 24 and standard deviation 9.25. Female respondents made up $52 \%$ of the sample (with $35 \%$ females at $\AA A \mathrm{AU}$ and $61 \%$ females at LU).

In Table 1 we illustrate how much the respondents are engaged in vigorous physical activity per week. In addition to vigorous exercise, we asked them about their participation in moderate or muscular physical training. Altogether we can conclude that most of the students are engaged in physical activities on a weekly basis. It is obvious that they are a potential target group for digital coaching. For more details, please see [26]. 
Table 1. Respondents vigorous physical activity on a weekly level

\begin{tabular}{|l|l|}
\hline Time in minutes per week & $\begin{array}{l}\text { Vigorous physical } \\
\text { activity } \mathrm{n}=137\end{array}$ \\
\hline $0 \mathrm{~min} /$ not at all & $13 / 9 \%$ \\
\hline$<30 \mathrm{~min}$ & $18 / 13 \%$ \\
\hline $30-60 \mathrm{~min}$ & $27 / 20 \%$ \\
\hline $60-90 \mathrm{~min}$ & $21 / 15 \%$ \\
\hline $90-120 \mathrm{~min}$ & $12 / 9 \%$ \\
\hline $120-150 \mathrm{~min}$ & $11 / 8 \%$ \\
\hline$>150 \mathrm{~min}$ & $35 / 26 \%$ \\
\hline
\end{tabular}

\subsection{Data processing for FsQCA}

To analyze the data using FsQCA, the following steps need to be performed on the original dataset: data calibration, truth table creation, assessing necessity relationships, identifying different solution sets (complex, parsimonious, intermediate), interpreting the results [20]. In the following we describe these main steps through our empirical dataset.

The variables included in the analysis with their corresponding construct are listed in Appendix 1. The first important task of FsQCA analysis is to transform variable values in order to have a range in the $[0,1]$ interval. According to the variables present in our dataset, the following transformations have been utilized:

- binary variables have been converted to assign 0 and 1 for the two possible values (for example gender in the data)

- variables measured with a scale of $n$ values have been transformed by converting any value $\mathrm{x}$ to $(\mathrm{x}$ 1) / n. An important point to note here is that in general the value of 0.5 plays an important role in the analysis as the cut-off value in later steps of the analysis. As in our further analysis each construct's final value is the average of several variable items, the value 0.5 rarely occurs in the final dataset used in the main steps of FsQCA.

A summary of the most important characteristic measures of the constructs used in the analysis are presented in Table 2.
Table 2. The descriptive statistics of fuzzy membership values (MS: mental support, EP: exercise program, G: goal setting, F: feedback, $S$ : social functionality, I: importance of digital coaching)

\begin{tabular}{|l|l|l|l|l|}
\hline Construct & Mean & Std. Dev & Min & Max \\
\hline MS & 0.64 & 0.27 & 0 & 1 \\
\hline EP & 0.56 & 0.20 & 0 & 1 \\
\hline G & 0.64 & 0.21 & 0 & 1 \\
\hline F & 0.61 & 0.22 & 0 & 1 \\
\hline S & 0.36 & 0.22 & 0 & 1 \\
\hline I & 0.69 & 0.23 & 0 & 1 \\
\hline
\end{tabular}

As a supplementary step, t-tests were performed focusing on the differences among respondents in Finland and Sweden. The only statistically significant difference in means was found in the construct Social Functionality (t-value $=-2.35, \mathrm{p}$-value $=0.02$ ): the Swedish respondents showed more interest in the social functions.

The next step of the analysis focuses on identifying causal conditions that are necessary for the outcome of interest to take place [24]. Finding an antecedent as necessary, as interpreting the meaning of the word in a fuzzy modelling context, would imply that whenever the outcome is present (high rating by a respondent), the attribute found as necessary should almost always be present (also rated highly by the respondent). In this initial step of the analysis the focus is on assessing the necessity of individual attributes and not on any complex configuration. An important reason for this (as we will see in the results presented below) is attributed to the fact that FsQCA is typically applied to complex real-life phenomena. In these problems, as already assumed by equifinality, one assumes that there are distinct ways to reach a given outcome. While this does not prevent a necessary condition to exist, in most studies researchers are not able to identify necessary conditions. Additionally, if we do not find any necessary individual conditions, we are guaranteed not to have any combination of attributes as necessary, as it would imply that some individual elements in the combination would have needed to be found necessary. As it is common, we performed the analysis for both the presence and absence of the outcome, trying to identify individual conditions that must be present for high/low ratings on 
the outcome variable. The results are presented in Tables 3 and 4, respectively. The strength of necessary relationships can be quantified using consistency and coverage measures. Consistency captures the required relationship between the input and output variable, with values higher than 0.9 indicating important relationships [22]. Coverage captures the importance of the relationship; the lower it is, the smaller is the number of cases to which the identified relationship is applicable.

As we can observe from the tables, while there are several values close to 0.9 , there is not a single attribute (or the absence of it) than can clearly be identified as necessary in order to observe consistently high or low ratings for the evaluation of digital coaching. With the notable exception of the social component, each attribute can be seen as more associated with the presence of the outcome variable rather than the absence of it.

Table 3. Necessary condition analysis for importance of digital coaching with the values in parenthesis evaluating the absence of the causal condition

\begin{tabular}{|l|c|l|}
\hline Construct & Consistency & Coverage \\
\hline MS & $0.88(0.45)$ & $0.87(0.85)$ \\
\hline EP & $0.76(0.55)$ & $0.94(0.86)$ \\
\hline G & $0.83(0.46)$ & $0.89(0.87)$ \\
\hline F & $0.79(0.50)$ & $0.89(0.88)$ \\
\hline S & $0.49(0.78)$ & $0.94(0.83)$ \\
\hline
\end{tabular}

Table 4. Necessary condition analysis for the absence of importance of digital coaching with the values in parenthesis evaluating the absence of the causal condition

\begin{tabular}{|l|c|c|}
\hline Construct & Consistency & Coverage \\
\hline MS & $0.83(0.72)$ & $0.41(0.62)$ \\
\hline EP & $0.80(0.88)$ & $0.45(0.62)$ \\
\hline G & $0.84(0.78)$ & $0.41(0.67)$ \\
\hline F & $0.85(0.78)$ & $0.43(0.63)$ \\
\hline S & $0.65(0.84)$ & $0.57(0.45)$ \\
\hline
\end{tabular}

\section{Results}

In this section we will present the results of the FsQCA analysis. The data preprocessing and analysis was performed utilizing different statistical and machine learning libraries of the $\mathrm{R}$ statistical programming language [19]. In the previous section we looked at the possibility of identifying necessary conditions of the perceived importance of digital coaching and as we have seen this is a strong requirement that is not satisfied fully by any of the possible causal antecedents. In the following we look at the sufficiency analysis to identify logical combinations of different subsets of the attributes that can be seen as causal explanations for the outcome of interest for a sufficiently large number of respondents in the sample.

In order to identify sufficient configurations, the first step is to construct the fuzzy truth table. In the truth table, all the possible combinations of antecedent predictor variables (including both presence and absence, i.e. high and low values). As in this study we have 5 variables, this potentially could result in $2^{\wedge} 5=32$ combinations, i.e. rows of the truth table. However, not all of the theoretically possible combinations actually occur in the sample, as it is normal unless an extremely large sample is taken. In our data, we found at least one corresponding respondent for 24 of the 32 possible configurations, with a large proportion (10) occurring only once. The three most frequently occurring configurations are the following:

- $\mathrm{MS}$ and $\mathrm{EP}$ and $\mathrm{G}$ and $\mathrm{F}$ and $\mathrm{S}$ (20 respondents)

- $\mathrm{MS}$ and $\mathrm{EP}$ and $\mathrm{G}$ and $\mathrm{F}$ and not $\mathrm{S}$ (42 respondents)

- not $\mathrm{MS}$ and not $\mathrm{EP}$ and not $\mathrm{G}$ and not $\mathrm{F}$ and not $\mathrm{S}$ (15 respondents)

Additionally to this analysis, we included gender (Ge), age (A) and education level (E) of the respondents as demographic variables. The next step in the analysis is to choose among the configurations that present in the data that conceivably contain relevant information. In order to do so, we need to select a threshold value on the number of occurrences of configurations in the data. As we have sufficient number of configurations with at least two corresponding respondents (and avoiding the presence of outliers in the form of unique individual opinions), we chose 2 as the cut-off threshold value. Additionally, for each configuration we need to determine whether it corresponds to the presence (high value, greater than 0.5 ) or absence (low value, smaller than 0.5 ) of the output variable. This can be captured by the consistency measure [20], for which any value above 
0.8 is acceptable. It is important to note that, as we will present it later, we performed the same analysis for (i) the presence of the output and (ii) the absence of the output; in both cases the critical value for frequency cutoff was set as 2, while for consistency we used:

- $\quad 0.97$ to identify configurations corresponding to the presence of the outcome

- $\quad 0.80$ to identify configurations corresponding to the absence of the outcome

The results of the analysis are presented in Tables 5 and 6 . In the tables, $\bullet$ indicates the presence and $\circ$ indicates the absence of a condition. With the exception of the condition gender, presence corresponds to high values and absence corresponds to low values. In case of gender, as per the arbitrary choice of our data coding, presence corresponds to females and absence corresponds to males. Empty cells indicate that the specific variable in that column is irrelevant for that configuration, i.e. irrespective of the value of that variable the configuration result in the presence of the output. From a methodological perspective, we can state that the solutions are sufficiently consistent in both cases and each rule has a meaningful coverage, i.e. not simply describe some individual extreme cases but characterize a sufficiently large proportion of the population.

Table 5. Solution configurations for the output variable set as the presence of the outcome, i.e. high perceived importance of digital coaching (Cov and

Con stands for Coverage and Consistency, respectively). The numbers in the first column refer to the five configurations.

\begin{tabular}{|l|l|l|l|l|l|l|l|l|l|l|}
\hline $\begin{array}{l}\text { Con } \\
f\end{array}$ & $\begin{array}{l}\text { G } \\
\text { e }\end{array}$ & A & E & MS & EP & G & F & S & Cov & Con \\
\hline P1 & & $\circ$ & & & $\bullet$ & & $\circ$ & & 0.48 & 0.96 \\
\hline P2 & $\circ$ & $\bullet$ & $\circ$ & & & & & & 0.20 & 0.95 \\
\hline P3 & $\bullet$ & $\bullet$ & & $\bullet$ & & & & & 0.16 & 0.98 \\
\hline P4 & $\bullet$ & $\bullet$ & & $\circ$ & $\bullet$ & $\bullet$ & & & 0.16 & 0.97 \\
\hline P5 & $\bullet$ & & $\bullet$ & & $\bullet$ & $\bullet$ & & & 0.39 & 0.97 \\
\hline
\end{tabular}

Table 6. Solution configurations for the output variable set as the absence of the outcome, i.e. no perceived importance of digital coaching (Cov and

Con stands for Coverage and Consistency, respectively). The numbers in the first column refer to the five configurations.

\begin{tabular}{|l|l|l|l|l|l|l|l|l|l|l|}
\hline Conf & $\begin{array}{l}\text { G } \\
\text { e }\end{array}$ & A & E & MS & EP & G & F & S & Cov & Con \\
\hline N1 & & $\bullet$ & $\circ$ & $\circ$ & $\circ$ & & & & 0.37 & 0.82 \\
\hline N2 & $\circ$ & & $\circ$ & & & & & & 0.32 & 0.79 \\
\hline N3 & $\circ$ & & & & $\circ$ & & & & 0.18 & 0.77 \\
\hline N4 & $\circ$ & $\bullet$ & & & & $\circ$ & & & 0.12 & 0.82 \\
\hline N5 & $\bullet$ & & & $\circ$ & & & & & 0.42 & 0.84 \\
\hline
\end{tabular}

It is important to note that individuals can be characterized by several of the configurations to some extent at the same time (as the consequence of fuzzy membership values), which is the reason why the coverage values sum up to a value greater than 1 , in contrast to probabilistic assignments.

\section{Discussion}

In this section the results of the FsQCA analysis are discussed and the derived configurations interpreted in line with our research objective. We start with some general observations, followed by the specific analysis of rules obtained for respondents with positive and negative perceived value of digital coaching.

Firstly, we can state that it is meaningful to apply FsQCA in this specific context as the two important assumptions discussed in our problem formulation seem to be not valid. We could not identify single (or combined) necessary conditions, moreover the resulting configurations illustrate the asymmetric nature of the problem: we cannot straightforwardly characterize respondents with negative outcome using the opposite of the rules obtained for respondents with positive outcome and vice versa. Moreover, the equifinality principle is clearly observable as we obtained several distinct configurations leading to the positive and negative outcome, with each of these rules having very strong consistency and covering significant fraction of the sample respondents. In each case, the analysis identified 5 important configurations characterizing different ways that lead a user to positively/negatively perceive digital coaching. 
Secondly, regarding individual antecedent variables, we can identify two that play (almost) insignificant role in understanding the outcome. The variable Social Functionality does not appear in any of the configurations. This is in line with the descriptive analysis identifying this variable as the one with the lowest mean value in our data. Still, it illustrates an important point that can be relevant to both academicians and practitioners, suggesting to attempt to focus more on other characteristics of digital coaching and their interplay with social functionalities. Additionally, the variable Feedback only appears in one of the 10 identified configurations, and even in that case low perceived importance of Feedback contributes to positive evaluation on digital coaching. While in this case most of the respondents rated Feedback highly as an individual component, the results show that it does not contribute to users' evaluation of digital coaching as a complete service.

After these general observations, we continue with looking at the configurations individually in Tables 5 and 6. The two set of configurations follow the same pattern with Gender of the respondents being a variable based on which they can be logically structured:

- one configuration that apply to both females and males (P1 and N1)

- $\quad$ several rules that characterize males $(\mathrm{P} 2, \mathrm{~N} 2, \mathrm{~N} 3$ and N4)

- $\quad$ several rules that characterize females (P3, P4, P5 and N5).

\subsection{Configurations for positive attitude towards digital coaching}

The first configuration, P1, identifies respondents who are of young Age, value Exercise program and do not value Feedback, and this is the most relevant rule with coverage value of 0.48 . Additionally to this configuration, the other rules can be grouped in a meaningful way based on the Gender variable. The first generic rule covers most of younger people, consequently most of the gender-specific rules include higher age as a component to offer a refined perspective on this age segment's behavior.

The single rule for males, P2, identifies a configuration that state that people with high Age who have low Education level value digital coaching. It is important to note at this point that as the data was collected from university students with a mean age of 28 years. This implies that when configurations identify respondents with high $\mathrm{Age}$, that means the age group of
28-35 years. For female respondents, the picture is more diverse. Females of higher Age perceive digital coaching positively as a result of two different possible ways:

- if they value Mental Support, that is a sufficient precondition (P3), or

- if they do not value Mental Support, then they value Exercise Program and Goal Setting as sufficient preconditions (P4).

The final rule for females, P5, states that if they have high Education level, value Exercise Program and Goal Setting, they will have a high perceived value of digital coaching. These findings show that Exercise Program and Goal Setting clearly have a positive effect for segments of the population, while Mental Support can be a positive or negative antecedent depending on other characteristics.

\subsection{Configurations for negative attitude towards digital coaching}

According to Table 6, the first configuration, N1, identifies respondents who are of high Age, low Education level, and do not value Mental Support and Exercise program as the most relevant general rule (coverage of 0.37). This confirms, although for a smaller portion of the population, the role of the two latter characteristics; while the presence of Mental Support and Exercise program seems to be an important facilitator, lack of them is clearly a negative one. In contrast to the previous table, here we have a more general explanation for respondents of high Age on why they do not positively perceive digital coaching. The same structuring based on the Gender variable also applies here, with the distinction that now we have three rules for males and a single rule for females.

The single rule for females, N5, identifies the absence of Mental Support as a sufficient precondition. This is probably the only rule for which we can find a symmetric corresponding configuration in the preceding section where we identified that the presence of Mental Support alone can be a sufficient precondition for positive evaluation of digital coaching. For male respondents, we have three straightforward rules:

- lack of Exercise Program results in general low perception of digital coaching (N2);

- male respondents with low Education level do not value digital coaching (N3); 
- males of high Age who do not think of Goal Setting as important, do not value digital coaching (N4).

These rules clearly show that by simply taking the complement of the configurations obtained for respondents with positive perception, we cannot obtain a good description of the respondents with negative opinion on digital coaching.

\section{Conclusions}

We present five configurations for respondents with high importance assigned to the digital coach, and five configurations to respondents with low assigned importance. Our results highlight the significance of exercise program and goal setting functionalities in a digital coach. The exercise program dimension appears in three of the configurations which correspond to the presence of the output variable - valuing digital coaching - one of them being the most relevant rule (P1). Goal setting appears in two such configurations. When looking at configurations related to the absence of our outcome variable, we can see that showing low interest in exercise programs and goal setting appear in three of the configurations; exercise program twice and goal setting once. Tailored exercise programs and suitable goal setting have been identified in previous research to be related to exercise adherence. Our results further stress the importance of designing digital coaching solutions with emphasis on individualized exercise programs and with meaningful, tailored goal setting functionalities.

The absence of feedback functionalities and social functionalities from the configurations related to the presence of the outcome variable warrants attention. Previous research highlights the importance of both feedback and social support in exercise participation and adherence [7, 8, 21]. Our respondents did value feedback as an individual dimension, but it does not seem to be important in the overall valuation of a digital coach. We can draw the conclusion that in our study, social functionality and feedback functionality does not play any role in the user's perception of the digital coach.

Some interesting gender-related observations arise. In our sample, three configurations assigning a high importance to the digital coach are related to the female gender; two of them showing interest in exercise programs and goal setting, one showing interest in mental support. It is not obvious from previous literature that there would be significant gender differences regarding e.g. exercise adherence, although [11] found some evidence that exercise planning might be more important for women than men, and [17] uncovered that positive reinforcement might be especially important for previously sedentary women. Our finding suggests that it is valuable to look into gender differences in the study and eventual design of digital coaching systems. Additionally, as we discussed and reflected on regarding the results in Table 5, users at the lower end of our age spectrum value digital coaching as captured in a single rule, while we need a larger number of configurations to understand the attitudes of users at the older end of the spectrum with confidence.

As this discussion illustrates, the use of FsQCA allowed us to obtain some novel insights that would have been difficult with traditional methods. First, as we clearly illustrated, the rules that explain negative attitude towards digital coaching are not simply the opposite of rules that explain positive attitude. This highlights the importance of considering the property of asymmetry as crucial in this domain, and with FsQCA we can straightforwardly handle this issue. Second, we clearly illustrated that the linearity principle does not hold for our dataset as it is not sufficient to look at the overall impact of an attribute in isolation from other attributes. As a clear example, depending on other attributes, both the presence of Exercise program (in configuration P5) and the lack of it (in configuration P2) can contribute to positive perception of digital coaching for different user groups. Third, the number of relevant configurations for both understanding positive and negative attitudes, illustrate the importance of considering the equifinality principle: different, distinct, rules can explain user attitudes for distinct user groups.

\section{A1. Appendix}

In the questionnaire, the respondents were asked to evaluate statements on a 5-point attitude scale based on the question 'How important do you think the following features are in a digital coach for physical training?'. The following are the statements used in the article listed under the corresponding construct:

\section{- MENTAL SUPPORT}

1. Helps you stay positive about yourself

2. Helps you stay focused on your goals

3. Helps you stay confident about yourself

\section{- EXERCISE PROGRAM:}

1. Designs a detailed exercise program for you

2. Gives you advice for proper warm-up 
3. Designs an exercise program that is suitably physically challenging

4. Designs an exercise program for a time period specified by you

5. Gives you advice on how to structure your training sessions

- GOAL SETTING

1. Helps you set specific goals

2. Helps you identify training strategies to achieve your goals

3. Monitors your progress towards goals

4. The digital coach helps you set target dates for attaining your goals

\section{- FEEDBACK}

1. Gives you specific, personalized feedback

2. Gives you feedback on your technique

3. Gives you positive feedback when you do things right

4. Gives you immediate feedback

- SOCIAL FUNCTIONALITY

1. The digital coach allows you to observe other users' training behavior

2. The digital coach allows you to compare your performance to other users' performance

3. The digital coach allows you to communicate with other users

4. The digital coach allows you to compete with other users

5. The digital coach gives you access to success stories of people who have reached their goals

6. The digital coach allows you to team up with other users to together reach your goals

The output(s) of the model were based on the following items, measured on a 4-point Likert scale:

- If I had access to such a digital coach, I would be interested in using it

- Such a digital coach would help me achieve my training goals

- Such a digital coach would help me stay motivated in my training

\section{References}

[1] Annesi, J. J., "Goal-setting protocol in adherence to exercise by Italian adults", Perceptual and Motor Skills, 94(2), pp. 453-458, 2002.
[2] Baker, J., J. Côté and R. Hawes, (2000). "The relationship between coaching behaviours and sport anxiety in athletes", Journal of Science and Medicine in Sport, 3(2), pp. 110-119, 2002.

[3] Bedrosian, R. C., R.H. Striegel-Moore and C. Wang, "Demographic and clinical characteristics of individuals utilizing an internet-based digital coaching program for recovering from binge eating", International Journal of Eating Disorders, 44(7), pp. 639-646, 2011.

[4] Chao, D., C.G. Foy and D. Farmer, "Exercise adherence among older adults: challenges and strategies". Controlled Clinical Trials, 21(5), S212-S217, 2000.

[5] Chari, S., A. Tarkiainen and H. Salojärvi, "Alternative pathways to utilizing customer knowledge: A fuzzy-set qualitative comparative analysis", Journal of Business Research, 69, pp. 5494-5499, 2016.

[6] Dam, L., D. Roy, D.J. Atkin and D. Rogers, D., “ Applying an integrative technology adoption paradigm to health app adoption and use", Journal of Broadcasting and Electronic Media, 62(4), pp. 654-672, 2018.

[7] Dubbert, P. M., A.D. Katell, J.K. Thompson, J.R. Raczynski, M. Lake, P.O. Smith, ... and R.E. Cohen, R. E., "Behavioral control of exercise in sedentary adults: Studies 1 through 6", Journal of Consulting and Clinical Psychology, 52(5), pp. 795-811, 1984.

[8] Duncan, S. C., T.O. Duncan and L.A. Strycker, "Sources and types of social support in youth physical activity", Health Psychology, 24(1), pp. 3-10, 2005.

[9] Felício, J. A., M. Duarte and R. Rodrigues, R., "Global mindset and SME internationalization: A fuzzy-set QCA approach", Journal of Business Research, 69, pp. 1372-1378, 2016.

[10] Fiss, P.C., "Building better causal theories: A fuzzy set approach to typologies in organization research", Academy of Management Journal, 54(2), pp. 393-420, 2011.

[11] Hankonen, N., P. Absetz, P. Ghisletta, B. Renner and A. Uutela, "Gender differences in social cognitive determinants of exercise adoption", Psychology and Health, 25(1), pp. 5569, 2010.

[12] Holmboe, E. S., M. Yepes, F. Williams and S.J. Huot, Feedback and the mini clinical evaluation exercise. Journal of General Internal Medicine, 19(5p2), pp. 558-561, 2004.

[13] Kettunen, E., T. Kari, M. Makkonen and W. Critchley, Digital coaching and athlete's self-efficacy: A quantitative study on sport and wellness technology, in Mediterranean Conference on Information Systems, MCIS, Springer, 2018. 
[14] Klaassen, R., R. op den Akker, T. Lavrysen and S. van Wissen, "User preferences for multi-device context-aware feedback in a digital coaching system", Journal on Multimodal User Interfaces, 7(3), pp. 247-267, 2013.

[15] Kulyk, O., R. op den Akker, R. Klaassen and L. van Gemert-Pijnen, L, "Personalized virtual coaching for lifestyle support: principles for design and evaluation", International Journal on Advances in Life Sciences, 6(3-4), pp. 300-309, 2014.

[16] Malhotra, R. and D.K. Malhotra, D. K., "Differentiating between good credits and bad credits using neuro-fuzzy systems", European Journal of Operational Research, 136, pp. 190-211, 2002.

[17] Noland, M. P., "The effects of self-monitoring and reinforcement on exercise adherence", Research Quarterly for Exercise and Sport, 60(3), pp. 216-224, 1989.

[18] Picorelli, A. M. A., L.S.M. Pereira, D.S. Pereira, D. Felício and C. Sherrington, "Adherence to exercise programs for older people is influenced by program characteristics and personal factors: a systematic review", Journal of Physiotherapy, 60(3), pp. 151-156, 2014.

[19] R Core Team, R: A language and environment for statistical computing. R Foundation for Statistical Computing, Vienna, 2018. URL: https://www.R-project.org/

[20] Ragin, C., Fuzzy-set social science, University of Chicago Press, Chicago, 2000.

[21] Rhodes, R. E., A.D. Martin, J.E. Taunton, E.C. Rhodes, M. Donnelly and J. Elliot, "Factors associated with exercise adherence among older adults", Sports Medicine, 28(6), pp. 397-411, 1999.

[22] Schneider, C. Q. and C. Wagemann, Qualitative Comparative Analysis (QCA) und Fuzzy Sets, Barbara Budrich, Opladen and Farmington Hills, 2007.

[23] Schneider, C. Q. and C. Wagemann, "Standards of good practice in qualitative comparative analysis (QCA) and fuzzysets", Comparative Sociology, 9(3), pp. 397-418, 2010.

[24] Schneider, C. Q. and C. Wagemann, Set-theoretic methods for the social sciences: A guide to Qualitative Comparative Analysis, Cambridge University Press, Cambridge, 2012.
[25] Sell, A., C. Walden and P. Walden, My wellness as a mobile app. Identifying wellness types among the young elderly. In Proceedings of the 50th Hawaii International Conference on System Sciences, 2017.

[26] Sell, A., P. Walden, C. Carlsson, M. Helmefalk, and L. Marcusson, Digital Coaching to Support University Students' Physical Activity, Proceedings of the 32nd Bled eConference, 2019, http://press.um.si/index.php/ump/catalog/book/418

[27] Smith, R. E. and F.L. Smoll, Social-cognitive approach to coaching behaviors, Social Psychology in Sport, pp. 75-90, 2007.

[28] Smithson, M. and J. Verkuilen, Fuzzy set theory: Applications in the social sciences (No. 147), Sage Publications Inc., 2006.

[29] Topman, R. M., D. Kruise and S. Beijne, Digital Coaching of Procrastinators in an Academic Setting, in H. C. Schouwenburg, C. H. Lay, T. A. Pychyl, and J. R. Ferrari (Eds.), Counseling the procrastinator in academic settings, pp. 133-148, American Psychological Association, Washington, DC, US, 2004.

[30] Veríssimo, J.M.C., "Enablers and restrictors of mobile banking app use: A fuzzy set qualitative comparative analysis (fsQCA)", Journal of Business Research, 69(11), pp. 5456$5460,2016$.

[31] Vis, B., "The comparative advantages of fsQCA and regression analysis for moderately large-N analyses", Sociological Methods and Research, 41(1), pp. 168-198, 2012.

[32] Wilson, K. and D. Brookfield, D, "Effect of goal setting on motivation and adherence in a six-week exercise program", International Journal of Sport and Exercise Psychology, 7(1), pp. 89-100, 2009.

[33] Yardley, J., J. Hay, W. Sedgwick and J. Baker, “An exploratory examination of the coaching behavior scale for sport", Avante, 5(3), pp. 82-92, 1999.

[34] Yueh, H.P., M.H. Lu and W. Lin, "Employees' acceptance of mobile technology in a workplace: An empirical study using SEM and fsQCA", Journal of Business Research, 69(6), pp. 2318-2324, 2016.

[35] Zadeh, L.A., "Fuzzy sets. Information and control", 8(3), pp. 338-353, 1965. 